\title{
Accuracy of Orthopedic Shoulder Test in Detecting Rotator Cuff Tears and Retears: A Narrative Review
}

\section{Brian H Goldman, DO (D) ${ }^{*}$, Alejandro Carmona-Casillas, BS ${ }^{2}$, Greg Galvin, DO ${ }^{1}$, Jorge Benito, DO ${ }^{1}$, Phong Truong, $\mathrm{DO}^{1}$ and Pedro J Tort-Saade, $\mathrm{MD}^{3}$}

\author{
${ }^{1}$ Department of Orthopedic Surgery, Larkin Community Hospital, South Miami, FL, USA \\ ${ }^{2}$ University of Medicine and Health Sciences, Basseterre, St. Kitts and Nevis, USA \\ ${ }^{3}$ Tort Orthopedic Institute, Professional Hospital, Guaynabo, Puerto Rico
}

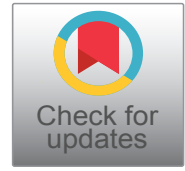

* Corresponding author: Brian H Goldman, DO, Department of Orthopedic Surgery, Larkin Community Hospital, 7000 SW $62^{\text {nd }}$ Ave, Suite 401, South Miami, FL 33143, USA, Tel: 305-284-7500

\begin{abstract}
The purpose of this review is to discuss the accuracy of orthopedic tests in detecting rotator cuff tears and retears, while developing new, more sensitive and specific clinical exam tools. With the ever-increasing population and consequent increased incidence of shoulder pathology, there is a vertical trend in medical cost as physical diagnosis is evermore substituted by imaging diagnosis. Unfortunately, clinical examination maneuvers have demonstrated variable specificity and sensitivity for primary rotator cuff tears, leading to the dependance of imaging modalities. Currently, there is no clinical exam maneuver that accurately confirms clinical suspicions of a rotator cuff retear following repair. Future studies focusing on the sensitivity and specificity of special orthopedic tests identifying retears would support a more accurate clinical diagnosis of rotator cuff retears. The goal of this narrative review is to outline the current trends in diagnosing rotator cuff tears and retears while demonstrating how current imaging studies have shown especially poor reliability in detecting this injury. This research project intends to present how a thorough and proper clinical exam that incorporates several orthopedic special tests, it can achieve an early and non-expensive accurate diagnosis.
\end{abstract}

\section{Keywords}

Rotator cuff tear, Retear, Arthroscopy, Orthopedic tests

\section{Abbreviations \\ US: Ultrasound; MRI: Magnetic Resonance Imaging}

\section{Introduction}

Over time, the incidence of rotator cuff repair has increased from about 23 to 83 people per 100,000 be- tween 1995 and 2009 and continues to increase [1]. An increased incidence of rotator cuff repair leads to an increase in diagnostic and pre-operative expenses in tools used for accurately diagnosing rotator cuff tears. In a study done in 2013, the PPAC (Per Person Average Cost) for diagnostic imaging for shoulder pain was $\$ 1,128$, from which $\$ 998$ were MRI (magnetic resonance imaging) expenses, \$88 for radiograms, \$33 for arthrograms and $\$ 20$ for additional imaging [2]. Diagnostic imaging is considered to be the gold standard for rotator cuff injuries, particularly MRI, but it has also proven to be highly expensive.

The incidence and prevalence of rotator cuff tears increase with age. The prevalence of cuff defects in patients over the age of 80 can reach upwards of $60 \%$ [3]. Although, many of these tears are initially asymptomatic, many studies have demonstrated that propagation of the defect increases, ultimately leading to pain in a large number of individuals.

Currently, only 3 physical exam findings described in the literature reliably assess rotator cuff pathology. The most sensitive test being the empty can test with $72 \%$ sensitivity [4], and the lift-off and belly-press tests which are poorly sensitive but very specific [5]. A study by Murrell, et al. suggested a combination of physical exam tests of supraspinatus weakness, external rotation weakness, and impingement tests could assist in the diagnosis of rotator cuff tears. When all three tests

Citation: Goldman BH, Carmona-Casillas A, Galvin G, Benito J, Truong P, et al. (2020) Accuracy of Orthopedic Shoulder Test in Detecting Rotator Cuff Tears and Retears: A Narrative Review. Int Arch Orthop Surg 3:016. doi.org/10.23937/2643-4016/1710016

Accepted: January 21, 2020; Published: January 23, 2020

Copyright: (c) 2020 Goldman BH, et al. This is an open-access article distributed under the terms of the Creative Commons Attribution License, which permits unrestricted use, distribution, and reproduction in any medium, provided the original author and source are credited. 
were positive, or if two tests were positive in a patient aged 60 or older, there was a $98 \%$ chance of having a rotator cuff tear [4].

\section{Rotator Cuff Retears}

The rate of rotator cuff retears following arthroscopic repair has been reported to vary in incidence from study to study as much as $9-94 \%$ and tends to occur within the first three months postoperatively [3,6-9]. One study in 2016 showed overall retear rate of $13.1 \%$ [3]. There were several significant variables. Symptom duration of 12 months or longer prior to having surgery was associated with a higher retear rate compared with a duration of symptoms shorter than 12 months (19.7\% vs. 8.5\%, respectively, $\mathrm{P}=0.006$ ) [3]. In addition, larger rotator cuff tears, defined using the Kim classification had the following retear rates: Type $D$ demonstrated $57.1 \%$ retear rate, type $C$ demonstrated $15.2 \%$, and type $A$ and $B$ demonstrated $8.0 \%(P<0.001)$ [3]. Also, tendon involvement significantly affected the retear rate: Combined supraspinatus, infraspinatus, and subscapularis tears had $42.9 \%$ rate of retear, compared to $10.0 \%$ in combined supraspinatus and infraspinatus tears, $22.2 \%$ in combined supraspinatus and subscapularis tears, and $6.6 \%$ with isolated supraspinatus tears $(P=0.001)$ [3]. A recent study published in 2018 reported an overall retear rate of $57.8 \%$ [10]. In a 10 -year study, up to $94 \%$ of patients at 2 -year follow-up after a rotator cuff repair demonstrated a recurrent defect, albeit with improved pain and outcome scores. Only 1 patient out of 18 in this study demonstrated no further arthropathy [11].

The risk for retears after arthroscopically repaired large rotator cuffs is multifactorial. This risk generally increases with age, with a retear rate as low as $5 \%$ in those under the age of 30, increasing significantly to $25 \%$ between the ages of 71 and 74 , and over $45 \%$ after the age of 85 [6]. Other predisposing factors responsible for high incidence of retear rates might be linked to factors such as smoking, hypercholesterolemia, and genetics [12]. However, the rates of retears may not necessarily be affected by age, sex, diabetes, smoking, or Goutallier classification. In fact, one study shows retear rates have been linked to duration of symptoms, position of the tendon relative to the glenohumeral joint, and tendon involvement. In this study, the overall retear rate was about $13.1 \%$ [3]. Another study published the most important independent prognostic factors predictive of a patient suffering retear of the rotator cuff after repair were degree of tendon retraction and acromion-humeral interval on preoperative X-rays [10].

Although rotator cuff retears are relatively common, there is still question as to the clinical significance in those with retears. Many studies, for instance, have shown no correlation between pain/functional outcomes and retears. Yoo, et al. reported a $45 \%$ retear rate after large rotator cuff repair with no clinical significance in outcome between those with a retear and those without [13]. Alternatively, other studies report poor clinical outcomes in those with retears. Mihata, et al. found poor outcomes in patients with failed massive rotator cuff repairs following single row, double row, and compression double row repair; reporting inferior scores for range of motion and active elevation in patients with retears compared to those with an intact repair [14]. A meta-analysis by Yang, et al. investigated these reported outcomes and found that patients with retears had lower clinical scores, such as UCLA and Constant Score, and decreased abduction strength [15].

\section{Orthopedic Shoulder Tests}

Clinical examination maneuvers with special tests have been described to assist in the diagnosis of a primary rotator cuff tear. These special maneuvers have demonstrated variable specificity/sensitivity for primary rotator cuff tears [16]. For primary supraspinatus tears, Jobe's test has a sensitivity/specificity of $88 \% / 62 \%$, Drop arm test has a sensitivity/specificity of $4 \% / 96 \%$, Hawkin's sign has a sensitivity/specificity of $64 \% / 48 \%$, and Neer's sign has a sensitivity/specificity of $60 \% / 58 \%$. For primary infraspinatus tears, Hornblower's sign has a sensitivity/specificity of $17 \% / 96 \%$ [16]. For primary subscapularis tears, lift off test demonstrates a sensitivity/ specificity of $22 \% / 94 \%$ [16]. The Rent test has demonstrated a sensitivity/specificity of $95.7 \% / 96.8 \%$ for partial and full thickness rotator cuff tears, with a diagnostic accuracy of $96.3 \%$ [17]. The Rent test has shown to be clinically favorable diagnosing rotator cuff tears compared to Jobe's, Hawkins and Neer's impingement signs and MRI [17]. However, there is a lack of analysis of these maneuvers in the aid of diagnosing rotator cuff retears following repair.

\section{MRI and US Diagnostic Accuracy in Primary Ro- tator Cuff Tears}

MRI and ultrasound (US) have classically been accurate tools for detecting primary full thickness tears. MRI detection of full thickness retear has demonstrated a sensitivity and specificity of $91 \%$ and $25 \%$, respectively [18]. A study by lannotti, et al. compared the accuracy between MRI (95\%) and US (88\%) to be comparable in diagnosing full thickness tears. However, the accuracy of partial thickness tears was less accurate between MRI and US with results of $73 \%$ and $70 \%$, respectively [19]. A systematic review by Lenza, et al. compared the diagnostic capabilities of US and MRI and determined that in the 11 studies that looked at either MRI or US detection of any rotator cuff tear, neither had superior statistically significant sensitivity or specificity. In summary, sensitivity and specificity of full thickness and partial thickness tears were $94 \%$ and $93 \%$, and $74 \%$ and $93 \%$ respectively, for MRIs. Sensitivity and specificity 
were $92 \%$ and $93 \%$ for full thickness tears, and $52 \%$ and 93\% for partial thickness tears when using US [7]. It was concluded that MRI and US both have generally lower sensitivities in detecting partial thickness tears than full thickness tears, with US only marginally better than chance in excluding partial rotator cuff tears [7].

Statistical findings from this systematic review demonstrate advanced imaging performed on 100 patients with shoulder pain (with an associated $80 \%$ prevalence of rotator cuff pathology), to have the following outcomes. There would be $3 \%$ and $9 \%$ missed rotator cuff tears and $20 \%$ and $15 \%$ wrongly detected rotator cuff tears with utilization of MRI versus US, respectively [7]. Although this only accounted for symptomatic patients as opposed to asymptomatic rotator cuff tears, this demonstrates the importance of performing a clinical exam in addition to imaging prior to performing surgery.

\section{MRI and US Diagnostic Accuracy in Rotator Cuff Retears}

Postoperatively, rotator cuff tendon can appear irregular in the first year and should not be confused for a retear. The lingering postoperative changes of increased signal intensity can represent granulation tissue and inflammation rather than a retear [20]. Additionally, portions of the original rotator cuff tear may not have integrated secondary to poor tissue quality and therefore would demonstrate features of a partial or full thickness tear, not to be confused for a retear. Accuracy aside, US demonstrates advantages over MRI for evaluation of rotator cuff repair, in settings where there are signal abnormalities due to suture, anchors, and excessive metal artifact or when $\mathrm{MRI}$ is contraindicated such as presence of a pacemaker [21,22].

In regard to retears, a study by Prickett, et al. found US to be accurate by correctly identifying 20 of 22 retears in patients who underwent rotator cuff repair, with a sensitivity and specificity of $91 \%$ and $86 \%$ [21]. Although the US detected all 3 partial thickness tears, this was only a small portion of the total of rotator cuff tears represented. Owen, et al. used MRI evaluation following rotator cuff repair which demonstrated an accuracy of $90 \%$ for detecting full thickness rotator cuff tears. Partial thickness tears were indistinguishable from repaired tendons [23]. A study by Roy, et al. compared MRI and US in the setting of retears and found both had sensitivities and specificities of $90 \%$ for full thickness tears but lower sensitivities for partial thickness tears ranging from 67-83\% [24]. Overall, full thickness retears are accurately detected using US and MRI, however both are far less accurate in detecting partial thickness rotator cuff tears.

Despite MRI and US demonstrating good accuracy in detecting full thickness rotator cuff retears, numerous studies have demonstrated that partial retears are easily missed. As previously mentioned, post-surgical changes seen on US and MRI imaging can make diagnostic interpretation challenging, particularly with MRIs. This is highlighted by Khazzam, et al.'s study on interobserver and intraobserver agreement of MRI imaging following rotator cuff repairs. After 7 fellowship trained orthopedic shoulder surgeons read 31 shoulder MRIs, the results demonstrated both intact and full thickness rotator cuff tears can be identified with moderate reliability [18]. This brings up the point that MRI and US imaging alone is not sufficient in dictating medical management. This further suggests that other imaging and clinical maneuvers need to be incorporated to improve diagnostic accuracy when there is suspicion of a retear.

\section{Conclusion}

Currently, there is no described clinical exam maneuver that accurately confirms clinical suspicions of a rotator cuff retear following repair. Although patients may present with preoperative risk factors or poor clinical outcomes associated with a retear, MRI and US imaging are almost exclusively relied upon to confirm a surgeon's suspicion for retear. Further development in diagnostic measures with new or adjunctive imaging modalities or new clinical exams would be beneficial. This is especially true given the challenges of deciphering postsurgical changes from a true tear as well as the variability of MRI and US accuracy in detection of retears, particularly partial thickness tear. Future studies analyzing the sensitivity and specificity of orthopedic special tests identifying retears would support a more accurate clinical diagnosis of rotator cuff retears.

\section{Author Contributions Statement}

Authors Chad Edwards, Brian Goldman, Andrew Hadeed, and Daniel Kalbac were involved in the following capacities:

- Substantial contributions to the conception or design of the work; Substantial contributions to the conception or design of the work; or the acquisition, analysis, or interpretation of data for the work; AND

- Drafting the work or revising it critically for important intellectual content; AND

- Final approval of the version to be published; AND

- Agreement to be accountable for all aspects of the work in ensuring that questions related to the accuracy or integrity of any part of the work are appropriately investigated and resolved.

\section{Conflicts of Interest}

We declare that we have no conflicts of interest in the authorship or publication of this contribution. 


\section{References}

1. Ensor KL, Kwon YW, Dibeneditto MR, Zuckerman JD, Rokito AS (2013) The rising incidence of rotator cuff repairs. J Shoulder Elb Surg 22: 1628-1632.

2. Yeranosian MG, Terrell RD, Wang JC, McAllister DR, Petrigliano FA (2013) The costs associated with the evaluation of rotator cuff tears before surgical repair. J Shoulder Elb Surg 22: 1662-1666.

3. Kim IB, Kim MW (2016) Risk factors for retear after arthroscopic repair of full-thickness rotator cuff tears using the suture bridge technique: Classification system. Arthroscopy 32: 2191-2200.

4. Murrell GAC, Walton JR (2001) Diagnosis of rotator cuff tears. Lancet 357: 769-770.

5. Yoon JP, Chung SW, Kim SH, Oh JH (2013) Diagnostic value of four clinical tests for the evaluation of subscapularis integrity. J Shoulder Elbow Surg 22: 1186-1192.

6. Diebold G, Lam P, Walton J, Murrell GAC (2017) Relationship between age and rotator cuff retear: A study of 1,600 consecutive rotator cuff repairs. J Bone Joint Surg Am 99: 1198-1205.

7. Le BT, Wu XL, Lam PH, Murrell GAC (2014) Factors predicting rotator cuff retears: An analysis of 1000 consecutive rotator cuff repairs. Am J Sports Med 42: 1134-1142.

8. McElvany MD, McGoldrick E, Gee AO, Neradilek MB, Matsen FA (2015) Rotator cuff repair: Published evidence on factors associated with repair integrity and clinical outcome. Am J Sports Med 43: 491-500.

9. Kim JH, Hong IT, Ryu KJ, Bong ST, Lee YS, et al. (2014) Retear rate in the late postoperative period after arthroscopic rotator cuff repair. Am J Sports Med 42: 2606-2613.

10. Shin YK, Ryu KN, Park JS, Jin W, Park SY, et al. (2018) Predictive factors of retear in cuff tear on shoulder MRI. AJR Am J Roentgenol 210: 134-141.

11. Paxton ES, Teefey SA, Dahiya N, Keener JD, Yamaguch $\mathrm{K}$, et al. (2013) Clinical and radiographic outcomes of failed repairs of large or massive rotator cuff tears: Minimum tenyear follow-up. J Bone Joint Surg Am 95: 627-632.

12. Tashjian RZ (2012) Epidemiology, natural history, and indications for treatment of rotator cuff tears. Clin Sports Med 31: 589-604.

13. Yoo JC, Ahn JH, Koh KH, Lim KS (2009) Rotator cuff integri- ty after arthroscopic repair for large tears with less-than-optimal footprint coverage. Arthroscopy 25: 1093-1100.

14. Mihata $T$, Watanabe $C$, Fukunishi $K$, Ohue $M$, Tsujimura $\mathrm{T}$, et al. (2011) Functional and structural outcomes of single-row versus double-row versus combined double-row and suture-bridge repair for rotator cuff tears. Am J Sports Med 39: 2091-2098.

15. Yang J, Robbins M, Reilly J, Maerz T, Anderson K (2017) The clinical effect of a rotator cuff retear. Am J Sports Med 45: 733-741.

16. Jain NB, Luz J, Higgins LD, Dong Y, Warner JJ, et al. (2017) The diagnostic accuracy of special tests for rotator cuff tear: The ROW cohort study. Am J Phys Med Rehabil 96: 176-183.

17. Wolf EM, Agrawal V (2001) Transdeltoid palpation (the rent test) in the diagnosis of rotator cuff tears. J Shoulder Elbow Surg 10: 470-473.

18. Khazzam M, Kuhn JE, Mulligan E, Abboud JA, Baumgarten $\mathrm{KM}$, et al. (2012) Magnetic resonance imaging identification of rotator cuff retears after repair: Interobserver and intraobserver agreement. Am J Sports Med 40: 1722-1727.

19. lannotti JP, Ciccone J, Buss DD, Visotsky JL, Mascha E, et al. (2005) Accuracy of office-based ultrasonography of the shoulder for the diagnosis of rotator cuff tears. J Bone Jointt Surg Am 87: 1305-1311.

20. Crim J, Burks R, Manaster BJ, Hanrahan C, Hung M, et al. (2010) Temporal evolution of MRI findings after arthroscopic rotator cuff repair. Am J Roentgenol 195: 1361-1366.

21. Prickett W, Teefey SA, Galatz LM, Calfee W, Middleton W, et al. (2003) Accuracy of ultrasound imaging of the rotator cuff in shoulders that are painful postoperatively. J Bone Joint Surg Am 85: 1084-1089.

22. Pierce JL, Nacey NC, Jones S, Rierson D, Etier B, et al. (2016) Postoperative shoulder imaging: Rotator cuff, labrum, and biceps tendon. Radiographics 36: 1648-1671.

23. Owen RS, lanotti JP, Kneeland JB, Dalinka MK, Deren JA, et al. (1993) Shoulder after surgery: MR imaging with surgical validation. Radiology 186: 443-447.

24. Roy JS, Braën C, Leblond J, Desmeules F, Dionne CE, et al. (2015) Diagnostic accuracy of ultrasonography, MRI and MR arthrography in the characterisation of rotator cuff disorders: A systematic review and meta-analysis. Br J Sports Med 49: 1316-1328. 